\title{
A New Approach to Inhibit Prototypic Galectins
}

\author{
Yves St-Pierre; Nicolas Doucet; and David Chatenet \\ Institut National de la Recherche Scientifique, INRS-Institut Armand-Frappier, Université du Québec, \\ 531 Boul. des Prairies, Laval, Québec, Canada, H7V 1B7 \\ FAX: +1-450-686-5501, TEL: +1-450-686-5354, E-mail: yves.st-pierre@iaf.inrs.ca
}

(Received on December 5, 2017, accepted on March 24, 2018)

Key Words: Prototypic galectins, galectin-7, dimer-interfering peptides, homodimers, immunotherapy

\begin{abstract}
Given the critical role of galectins in cancer and other diseases, considerable efforts have been deployed towards the development of carbohydrate-based inhibitors that limit the binding of galectins to glycosylated residues on cell surface receptors. However, despite decades of research, progress in this field has not met expectations. In this article, we discuss the rationale justifying the development of a new class of galectin-specific peptide inhibitors that disrupt the formation of a prototypic galectin and its protumorigenic functions. These dimer interfering peptides (DIPs) represent an interesting alternative-and possibly a complementary avenue - to neutralize galectin-mediated protumoral functions. If validated, the approach could broaden the classes of galectin inhibitors that can be readily generated against other prototypic galectins, and possibly all other galectin subtypes.
\end{abstract}

\section{A. The Need for New Galectin Inhibitors}

Members of the galectin family exert essential functions by interacting with a large diversity of binding partners, both outside and inside cells. Following their release outside cells, either passively from dead cells or actively via non-classical secretion pathways, extracellular galectins bind glycosylated growth receptors on the surface of normal and cancer cells to control their signaling threshold. These interactions are primarily mediated by a highlyconserved glycan binding site (GBS) located on the surface of each carbohydrate recognition domain (CRD) that form galectin dimers or oligomers (Fig. 1A-B). However, under stress or pathological conditions, the expression level of galectins is often significantly increased and leads to misregulated functions. For example, increased expression of intracellular galectins in cancer cells may lead to altered cell growth, sensitivity to chemotherapeutic agents, and/or increased invasive behavior (1). Outside the cell, galectins bind to glycosylated receptors on the surface of immune cells to control the fate of effector and regulatory lymphoid and myeloid cell populations (2). Such properties enable galectins to induce apoptosis of cancer-killing $\mathrm{T}$ cells or to induce a tolerogenic state in tumor-associated macrophages (3). Thus, galectins represent a significant obstacle to successful cancer immunotherapy. Considerable efforts are therefore being deployed to modulate their activities. Until now, most efforts have focused on carbohydrate-based inhibitors aimed at disrupting GBS-dependent intracellular signals triggered upon binding of galectins to cell surface glycoreceptors. Often, these inhibitors are high molecular weight, naturally occurring or chemically-modified plant polysaccharides with significant structural complexity, or small mono/disaccharides that block binding of extracellular galectins to cell surface glycoreceptors $(4,5)$.
These include modified citrus pectin (MCP) or GCS-100. In some cases, the effectiveness of this approach in neutralizing the galectin-mediated immune suppression has been demonstrated using in vivo preclinical studies. For example, intratumoral injection of thiodigalactoside (TDG) increases the infiltration of cancer-killing immune cells $(6,7)$. However, the effectiveness of plant oligosaccharides and other galectin carbohydrate-based inhibitors in restoring cancer-killing activity of immune cells, such as the synthetic glycoamine analog lactulosyl-L-Leu, remains to be established. Until now, studies on the anti-tumoral efficacy of these compounds have mostly focused on their ability to inhibit the survival of human cancer cells or their resistance to drug-induced apoptosis (8-10). Moreover, these carbohydrate-based inhibitors often have a relatively low binding affinity for galectins and the assumption that many of these drugs are true selective CRD inhibitors has recently been challenged (11).

Based on the hypothesis that galectin ligand binding avidity is increased during clustering of glycoreceptors, several investigators have thus developed synthetic glycopolymers/glycodendrimers that target the GBS of galectins (12-15). Yet, the greatest challenge using GBS inhibitors (GBSIs) is to achieve high selectivity to minimize off-target effects, especially considering the striking structural and GBS similarity between all human galectin CRDs (Fig. 1C). The use of (glyco)peptides identified following screening of libraries is an interesting avenue that is being explored to identify GBSIs with better selectivity for galectin-1 and -3 (16). High selectivity is a critical issue because carbohydrate-based inhibitors with limited selectivity may also have limited efficacy given the pro- and anti-tumoral functions of galectins and the relatively wide repertoire of galectins expressed in tumor tissues. We and others 


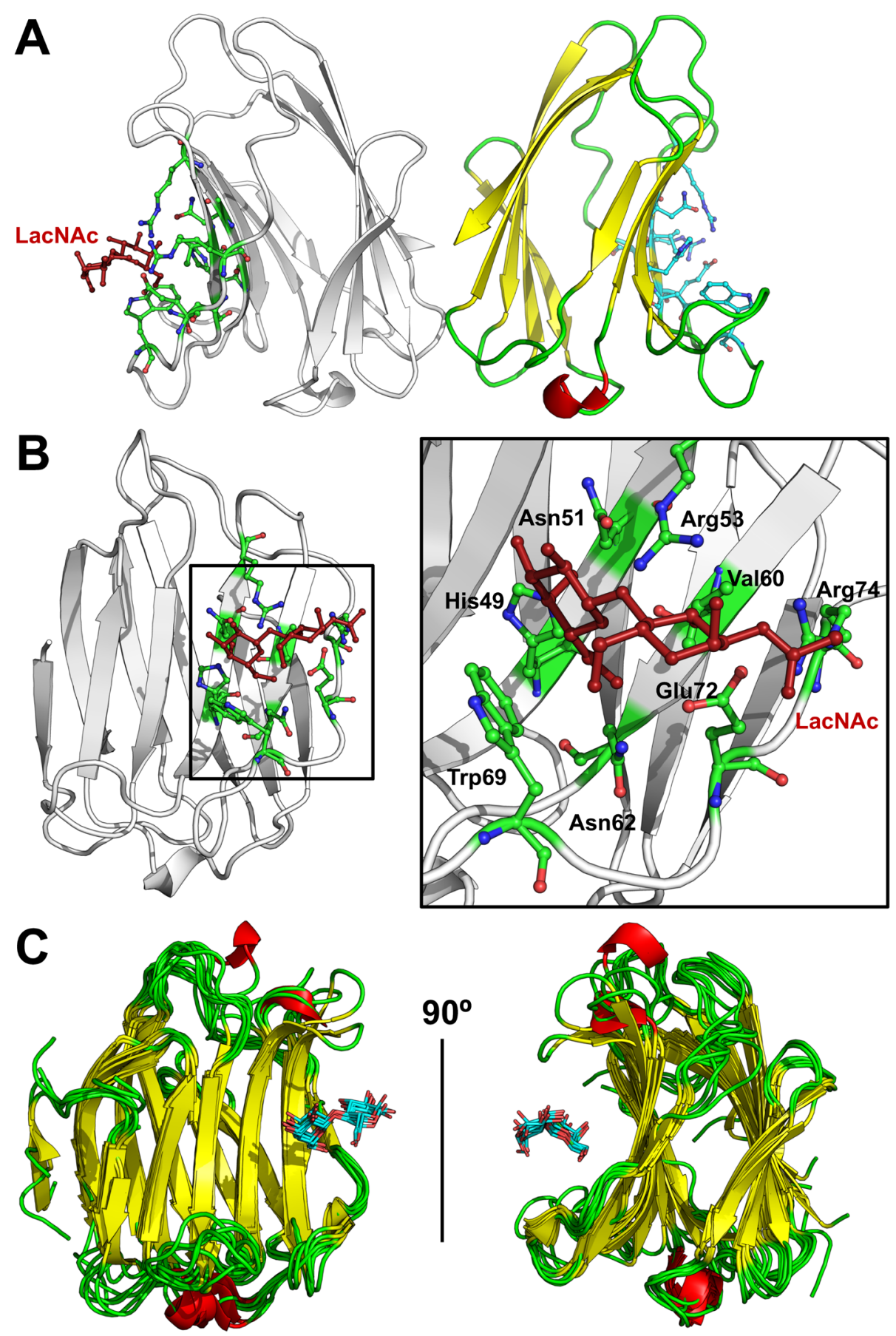

Fig. 1. Representative homodimeric structure of a prototypic galectin. A) Crystal structure of human galectin-7 showing the $30 \mathrm{kDa}$ 'back-toback' homodimer architecture of the two carbohydrate recognition domains (CRDs) (PDB entry 5GAL). Protomer 1 (gray, on the left) is complexed with disaccharide $N$-acetyllactosamine (LacNAc, in red), sitting in the glycan binding site (GBS). Residues of the GBS are shown as ball-and-stick representation with standard coloring scheme: red for oxygen, blue for nitrogen, and green for carbon. Protomer 2 (on the right) is unbound and colored by secondary structure: yellow for $\beta$-strands, red for $\alpha$-helix, and green for random coil. B) Side-view of protomer $1\left(90^{\circ}\right.$ counter clockwise rotation about the $\mathrm{y}$-axis relative to A), seen from the concave side that binds carbohydrate ligands. Each galectin-7 protomer is $15 \mathrm{kDa}(135 \mathrm{residues})$ and forms a $\beta$-sandwich secondary structure with two slightly bent $\beta$-sheets delineating a convex and a concave side. The boxed area illustrates the glycan binding site (GBS), zoomed on the right. Highly conserved residues involved in hydrogen bonding, hydrophobic, polar, and electrostatic interactions with LacNAc and/or important crystallographic water molecules are shown as ball-and-stick representation. C) Overlay of all structurally resolved monomeric human CRDs from GAL-1, -2, -3, -4, -7, -8, -9, -10, with lactose-bound ligands shown as cyan sticks (PDB entries 1QKQ, 2EAK, 5GAL, 3AP4, 1W6O, 4R9A, 4YM3, and 5DG2). Striking structural similarity between CRDs, analogous ligand positioning in the glycan binding site, and highly conserved GBS residues further illustrate that individual galectin specificity is not exclusively controlled by their carbohydrate binding lectin properties. Galectin function is also mediated by distinct oligomeric architectures and glycan-independent interactions with small-molecule and/or protein partners. 
have shown that breast and prostate cancer tissues express multiple galectins that contribute to the heterogeneity of cancer. Using tissue microarrays constructed with biopsies of breast cancer patients, we found that multiple galectins, including GAL-1, $-3,-7,-8$, and -9 , were expressed across all molecular subtypes of breast cancer, except for GAL-7, which was specifically expressed in aggressive Her-2-positive and triple-negative subtypes $(17,18)$. Yet, the field of galectin inhibitors has been largely dominated by studies that almost exclusively focused on GAL-1 and GAL-3. We still have very limited knowledge of the role of other galectins in mediating immune functions, and whether they exhibit pro- or anti-tumoral properties. A good example of such contradictory role for galectins is provided by our data in breast cancer. We have shown that while GAL-1 is associated with cancer progression, GAL- 8 has a protective effect (18). Also, in colon cancer, while GAL-1 and GAL-7 are pro-tumorigenic, GAL-4 acts as a tumor suppressor (19-21). Such dual role for galectins in cancer is well documented, especially for GAL-3 and GAL-7 (22-24). A better knowledge of the fine specificity contributions of the GBS and the rational design of drugs that take into account these fine specificities may help towards a successful drug development path for GBSIs. Another challenge in the development of galectin inhibitors is that GBSIs are not effective at targeting glycan-independent functions of galectins. As discussed below, accumulating evidence shows that several biological functions of galectins are accomplished through glycan-independent interactions. Consequently, there is an urgent need to go back to the drawing board to refine our strategies for the development of new, effective, and specific galectin inhibitors.

\section{B. Glycan-Independent Functions of Galectins?}

For a long time, plant lectins have been known to bind noncarbohydrate and hydrophobic ligands such as peptides, adenine, fluorescent probes, and porphyrins in a glycan-independent fashion, often exhibiting higher affinities than their 'natural' saccharide ligands (26). Unsurprisingly, the numerous biological functions of galectins can also be accomplished independently of their lectin activity. This has been well documented for intracellular galectin activities. For example, GAL-7 interacts with intracellular BCL-2 family members via a GBS-independent interaction $(26,27)$. Similarly, intracellular GAL-3 and -4 directly bind to beta-catenin, a non-glycosylated protein $(28,29)$. Additionally, GBS-independent functions of galectins are not limited to their intracellular activities. This is particularly well illustrated during the formation of an immune synapse following the binding of GAL-1 to the surrogate light chain (SLC) of the pre-B cell receptor, whereby GAL-1 is produced by the stromal cells from the bone marrow (pre-BCR) complex expressed on the surface of immature B cells (30). GAL-1 directly binds to a non-glycosylated form of SLC, indicating a direct protein-protein interaction. A GBS-independent mechanism was also observed during the activation of glial cells by extracellular soluble GAL-3 (31), or activation of human monocytes by GAL-2 (32). Clathrin-mediated endocytosis of GAL-3 by M1 macrophage is also carbohydrate-independent and mediated by its $\mathrm{N}$-terminal domain, as evidenced by its insensitivity to lactose or TDG139, or to mutations that render its GBS ineffective to lactose binding (33). GAL-3 also binds to bacterial polysaccharides (LPS) via a non-GBS-mechanism (34). From an evolutionary perspective, such GBS-independent function in galectins may not be so surprising. Many galectin-like proteins in mammals do not exhibit $\beta$-galactoside binding activity, including the lens crystallin protein GRIFIN (galectin related inter-fiber protein), which forms a stable homodimer, and the galectin-related protein GRP (previously known as HSPC159; hematopoietic stem cell precursor), which exists as a monomer in solution (35-37). In zebrafish and chicken, however, the GRIFIN homolog can bind $\beta$-galactosides $(38,39)$, suggesting that galectin-like proteins have evolved from ancestral galectins to accommodate a variety of protein-protein interactions with cell surface receptors to modulate intracellular signals.

Our recent findings in prostate cancer showing that alterations in the GBS of GAL-7 shift the balance towards GBS-independent binding partners and drive a phenotypic switch in cancer cells emphasize the importance of GBS-independent galectin activity in cancer (40). Apart from GAL-3, which mediates protein-protein interactions via its long polypeptide tail, we know very little about how prototypic and/or tandem-repeat galectins mediate GBS-independent interactions. For prototypic galectins, which are typically found in a monomer/homodimer equilibrium in solution, it is logical to hypothesize that GBS-independent interactions may involve residues located at the homodimeric interface. This equilibrium may depend on galectin concentrations within a specific cellular compartment, at least in the case of GAL-7, as suggested by data from a study by Ermakova et al. (41). Using FPLC gel filtration experiments, the authors showed that lactose-free GAL-7 was mostly found as a homodimer at high $(>100 \mu \mathrm{M})$ concentrations and as a monomer at lower concentrations. They also found that the binding of lactose shifts this equilibrium towards a more stable homodimer form, a conclusion supported by their circular dichroism-based thermal denaturation studies. Their NMR analysis further showed that GBS occupancy possibly promotes reorientation of residues at the dimer interface. This suggests that occupancy of the GBS with endogenous ligands could potentially impact protein-protein interactions, which in turn may rely on fine specificities of the GBS. This has been shown for GAL-9, a member of the tandem repeat subtype of galectins (42). For prototypic galectins, 
comparative structural studies using NMR spectroscopy, isothermal titration calorimetry, and X-ray crystallography of their CRD in the free state and in complex with protein ligands will be needed to provide a detailed understanding of how ligand binding to the GBS affects protein-protein interactions and galectin architecture, and inversely whether such interactions modulate the fine specificities of the GBS. Solving these issues could lead to a paradigm shift in our understanding of galectin function and the development of galectin-specific antagonists.

\section{Galectin Oligomerization: an Evolutionary Per- spective}

Like most proteins belonging to the lectin family, galectins harbor a tertiary structure characterized by a 'jelly roll' fold consisting of anti-parallel $\beta$-sheets of approximately 135-140 amino acid residues (Fig. 1). The 3D structure of each protomeric CRD is similar to the jelly roll CRD found among plant and other ancestral lectins. Ancestral lectin structural organization further suggests that family members have gone through selective pressure for stabilizing a multivalent quaternary structure to increase ligand affinity and/or to promote a distinct biological activity. This occurs either following the association of different mono- or dimeric structures,
GAL-1

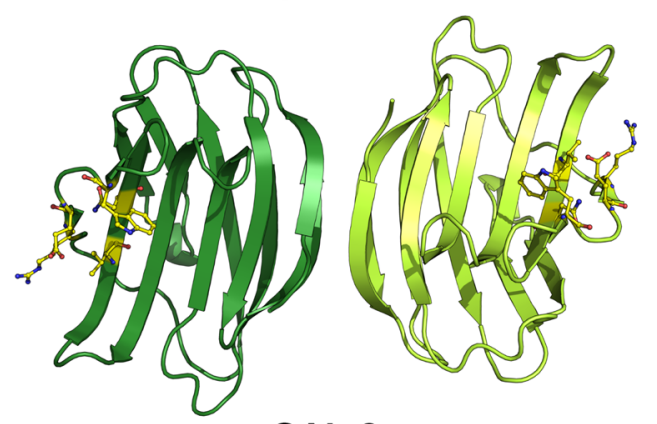

GAL-2
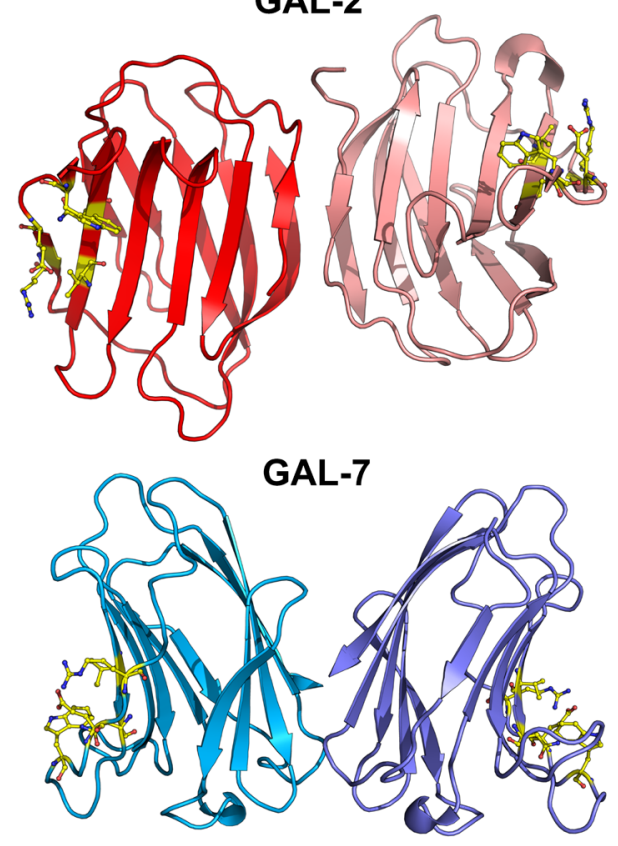

CchG-1
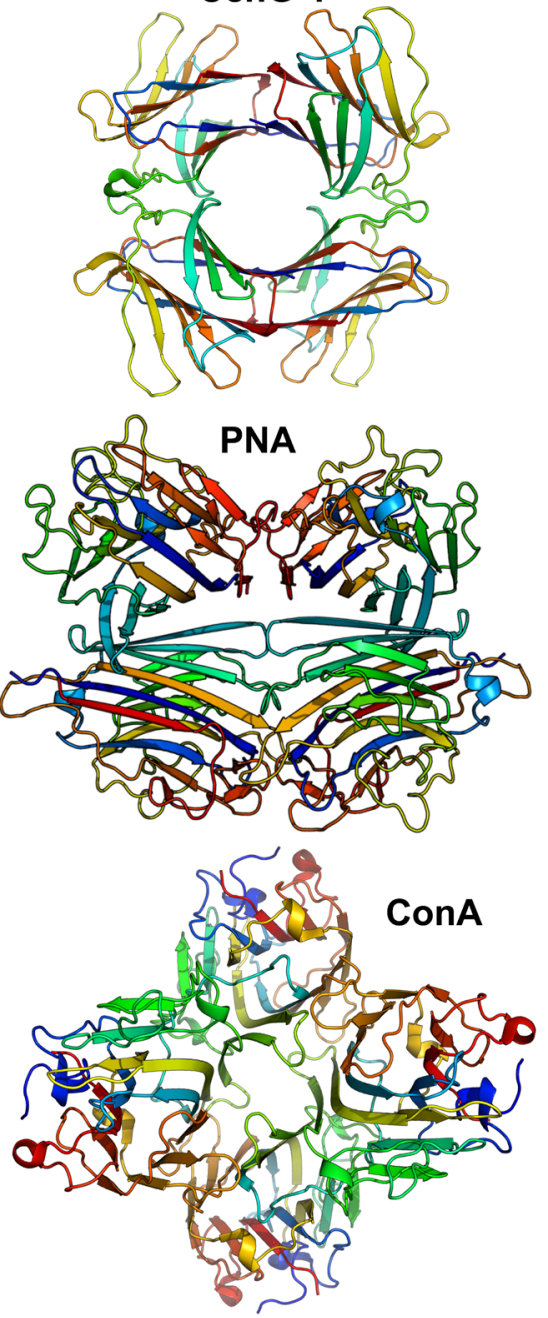

Fig. 2. Oligomerization of lectins and galectins through evolution. Lectins and galectins are found in nearly all life forms and exhibit similar jelly roll carbohydrate recognition domains (CRDs), but distinct quaternary structural architecture. The dimeric structure of prototypic human galectins can display 'side-by-side' CRD homodimerization (GAL-1 and GAL-2, PDB entries 3W58 and 5DG2), or 'back-to-back' homodimerization (GAL-7, PDB entry 5GAL). Tetramer formation is often observed in plant and ancestral lectins, with significantly distinct packing architecture. Tetramer architectures are shown for the CchG-1 galectin from Cinachyrella sp. (Ball Sponge, PDB entry 4AGV), the PNA lectin from Arachis hypogaea (Peanut Lectin, PDB entry 1CIW), and concanavalin A from Canavalia ensiformis (Jack Bean, PDB entry 1CJP). Highly conserved residues of the GBS are shown on galectins as ball-and-stick representation with standard coloring scheme: red for oxygen, blue for nitrogen, and yellow for carbon. 
or via multiple CRDs encoded within a single polypeptide chain. Such multivalency is a common feature of lactose- and galactosebinding lectins, which can form tetramers, hexamers, and octamers through a variety of quaternary arrangements and subunit swaps (Fig. 2) $(25,43,44)$. For example, the oyster Crassostrea virginica expresses two tetrameric galectins (cvGAL-1 and cvGAL-2), consisting of four tandemly arrayed CRDs that help recognize extrinsic carbohydrates on the surface of potentially pathogenic infectious agents, and to initiate their phagocytosis and elimination by circulating hemocytes $(45,46)$. cvGAL- 1 is encoded by a single copy gene containing 12 exons and encoding a 560-amino acid-long polypeptide with four similar but distinct CRDs that are linked together by 8-17 residue long linker peptides. In the case of cvGAL-2, its polypeptide is encoded by a similar gene organization consisting of 13 exons, giving rise to a 557-residue protein with a calculated molecular weight of $64.1 \mathrm{kDa}(46)$. Like galectins from mammals and other species, both cvGAL-1 and cvGAL-2 are released in the extracellular space via an unconventional secretion mechanism, allowing them to fulfill their various immune functions. Their oligomeric structure allows for cooperative binding and confers high avidity for the target, a property that is believed to be profitable for recognition and neutralization of infectious pathogens. Genes exhibiting high primary structure homology to cvGAL-1 and cvGAL-2 have also been found in another closely related oyster species, Crassostrea gigas (46). Such tetrameric structure for galectins is not unusual and has been found following analysis of RNA-Seq data available for the blue mussel Mytilus galloprovincialis (47) and many other bivalves and gasteropods (48). It has also been found in the genome of Lingula anatina, the most primitive group of brachiopods known to have existed since the Cambrian (49). This GAL-4-like protein consists of four covalently linked CRDs forming a 576-residue polypeptide encoded by a 2331 nucleotide mRNA. Another galectin from a family of novel 'prototype' galectins from a Japanese marine sponge, Cinachyrella sp. (CchG-1), consists of a homotetramer with a central 'donuthole' surrounded by four $16-\mathrm{kDa}$ protomers $(50,51)$ (Fig. 2). This oligomeric architecture for a galectin from an ancestral organism has also been recently described in the case of CGL, a lectin from the sea mussel Crenomytilus grayanus (52). Although we still ignore whether CGL exists as a monomer or oligomer in living organisms, crystal structures revealed a hexameric organization consisting of six CGL protomers. This lectin is structurally close to MytiLec, another recently described lectin with $85 \%$ identity to CGL found in the blue mussel, Mytilus galloprovincialis $(52,53)$.

What controls the equilibrium between monomeric and oligomeric forms of galectins in living organisms remains poorly understood. Except for GAL-3, which forms oligomers via at least two distinct mechanisms of action (54), in general, for prototypic galectins, it is believed that this equilibrium is modulated, at least in part, by galectin concentration, although this assumption has been challenged in the case of GAL-1 (55). In the case of xgalectinVa from Xenopus laevis, a galectin that forms (homo)dimers and (homo)tetramers in solution (56), oligomerization is promoted by lactose binding, thereby facilitating aggregation of non-self-infectious pathogens while reducing the accessible molecular surface to prevent non-specific aggregation (57). Altogether, these studies in invertebrates reveal that oligomerization of galectin CRDs is beneficial for their ability to carry out their roles as innate sensors and extracellular immune effectors in response to bacterial invasion. The benefits of such oligomers on the ability of a protein to bind multiple cell surface receptors and to maximize its ability to trigger receptor-induced signaling has been hypothesized in many systems, such as the binding of human MHC class II to the T cell receptor $(58,59)$. In the case of human lectins, such multivalency does indeed help reorganize the distribution of cell-surface glycoreceptors into clusters or lattices $(60,61)$. Such redistribution of glycoreceptors modulates the delivery of intracellular signals that regulate various cellular functions, including cell proliferation, cytoskeletal reorganization, gene expression, and cell survival. For galectins, the importance of oligomerization has been particularly well studied in the case of GAL-3, which forms oligomers via its $\mathrm{N}$-terminal peptide when bound to cell surface glycoreceptors (62$65)$. Interfering with this multivalency is thus a potentially worthy, yet poorly explored, strategy to inhibit immunosuppressive functions of galectins when they bind glycoreceptors on cancer-killing lymphocytes.

\section{Analysis of the Prototypic Galectin Homodimer In- terface}

Although lectins generally exhibit similar monomeric 3D structures in their $\mathrm{CRD}$, their distinctive quaternary architecture has recently attracted considerable interest due to the biological significance of unique molecular determinants defining their oligomeric organization. This is especially true for prototypic galectins, the major subfamily type found in humans and other species (66). In mammals, from the 15 galectins that have been identified to date, 9 are prototypic (GAL-1, -2, -5, -7, -10,-11, -13, -14, and -15). Except for GAL-5, which is exclusively monomeric and has only been found adsorbed on the surface of rat reticulocytes and erythrocytes $(67,68)$, prototypic galectins adopt a homodimeric quaternary structure. How lectins form homodimers has been the subject of intense research. In some cases, such as plant lectins, specific signature sequences have been identified to explain the different modes of association implicated in the formation 
of homodimers (69). Each human galectin has its own sequence signature, which excludes most of the residues found in the signature sequence of the plant lectin interface. In fact, examination of human GAL-1, GAL-2, and GAL-7 reveals that each has its own homodimerization pattern. While GAL-1 and GAL-2 respectively adopt 'side-by-side' and twisted 'side-by-side' orientations of their CRDs, GAL-7 exhibits a 'back-to-back' CRD homodimerization architecture (Fig. 2) (44). The homodimeric interface of human GAL-7 is estimated to cover a surface of approximately $1494 \AA^{2}$, as compared to $1093 \AA^{2}$ and $1179 \AA^{2}$ for GAL-1 and GAL-2, respectively (70). The larger homodimeric interface of GAL-7 is stabilized by a significant number of electrostatic interactions between symmetrical arginine, lysine, aspartate, and glutamate residues on opposite protomer subunits (Fig. 3A-B). Protected by these numerous electrostatic interactions, a solvent-shielded hydrophobic core of leucine, isoleucine, valine, and phenylalanine residues lies within this homodimeric interface. The homodimeric interface of other galectins is also stabilized by similar non-bonded interactions. However, since most 'side-by-side' homodimers primarily rely on a smaller number of hydrogen-bonding interactions involving 4 antiparallel $\beta$-strands, they may generally be weaker than those observed in the 'back-to-back' dimer orientation of
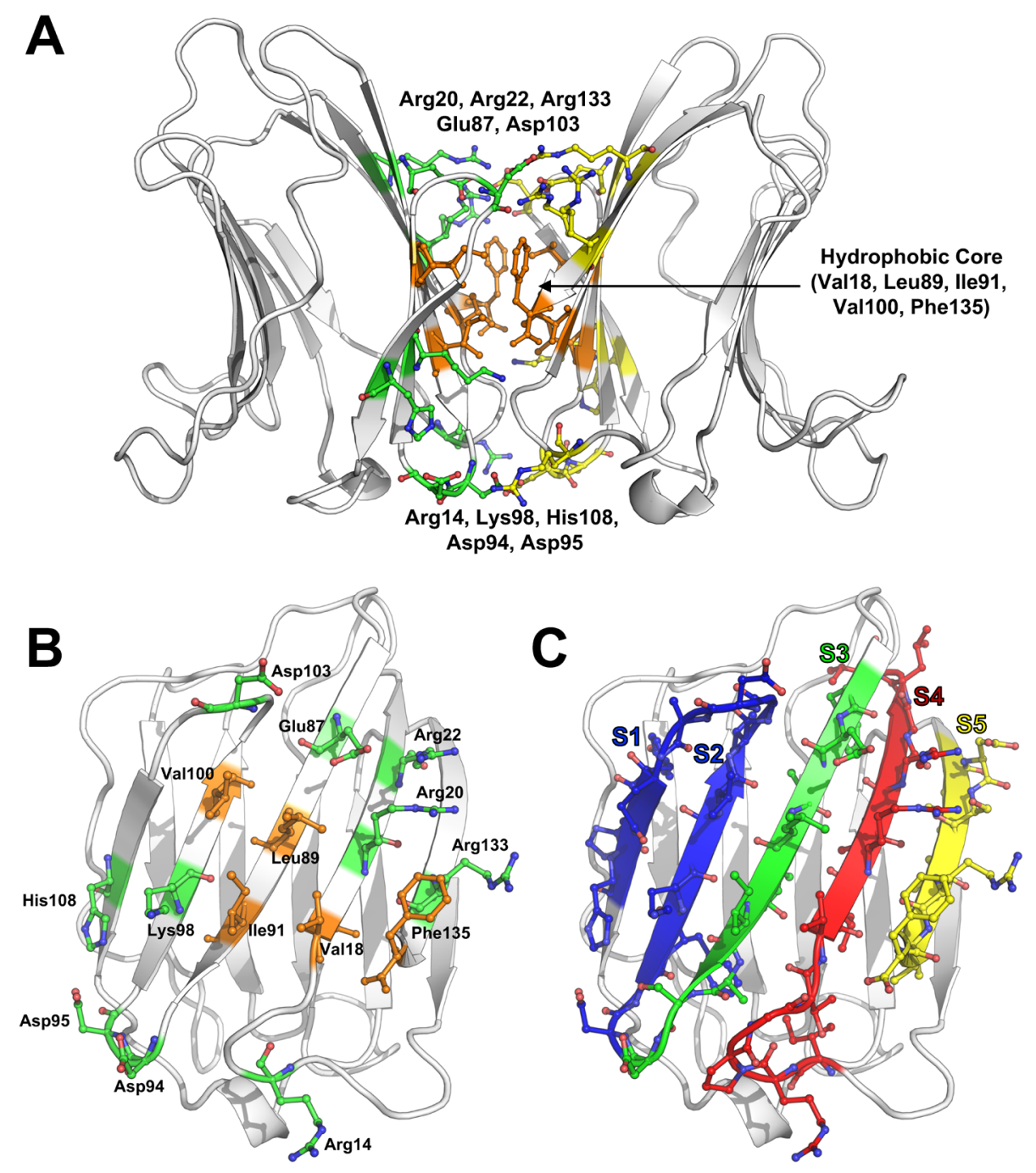

Fig. 3. Rational design strategy for selecting GAL-7 dimer interfering peptides (DIPs). A) Electrostatic and hydrophobic interactions stabilizing the 'back-to-back' homodimer interface of GAL-7. Polar and charged residues sit above and below the hydrophobic core of the interface. Residues are shown as ball-and-stick representation with green or yellow carbon coloring for residues from protomer 1 (left) and protomer 2 (right), respectively. B) Side-view of protomer 1 ( $90^{\circ}$ clockwise rotation about the y-axis relative to A), showing individual residues involved in the GAL-7 interface. C) To disrupt the homodimer interface of GAL-7, mimic peptides encompassing residues 13-25 ( $\beta$-strand S4, red), 86-102 ( $\beta$-strands S2S3, green), 95-108 ( $\beta$-strands S1-S2, blue), and 129-135 ( $\beta$-strand S5, yellow) were synthesized and tested for their ability to disrupt GAL-7 homodimerization and biological function [Vladoiu et al., 2015]. Due to sequence overlap between designed peptides 86-102 and 95-108, only $\beta$-strand S3 of peptide $86-102$ is colored green on the structure. 
GAL-7. In fact, a single mutation at the extreme N-terminus of GAL-1 is sufficient to alter the monomer-dimer equilibrium (71). Naturally, the residues involved are completely distinct from one galectin to the other, and so is the architecture formation of the dimer interface (Fig. 2). Such differences render them incapable of forming a GAL-7-like interface, further providing an interesting target area to generate inhibitor selectivity via specific signature sequence motifs.

\section{E. Disruption of Galectin-7 Homodimers}

As a proof-of-concept for testing whether disruption of the homodimer interface of galectins is a viable strategy for developing effective and selective inhibitors, our group has examined the dimer interface region of human GAL-7, which behaves as a dimer in solution and also crystallizes as a dimer $(70,72,73)$. We focused on GAL-7 because our previous work and that of others showed that it is the only galectin family member with an expression profile restricted to epithelial cells. Abnormally high expression of GAL-7 is also associated with aggressive subtypes of many carcinomas, including breast cancer, for which there are limited therapeutic options. Moreover, we have previously shown that GAL-7 plays a pivotal role in controlling the metastatic behavior of breast
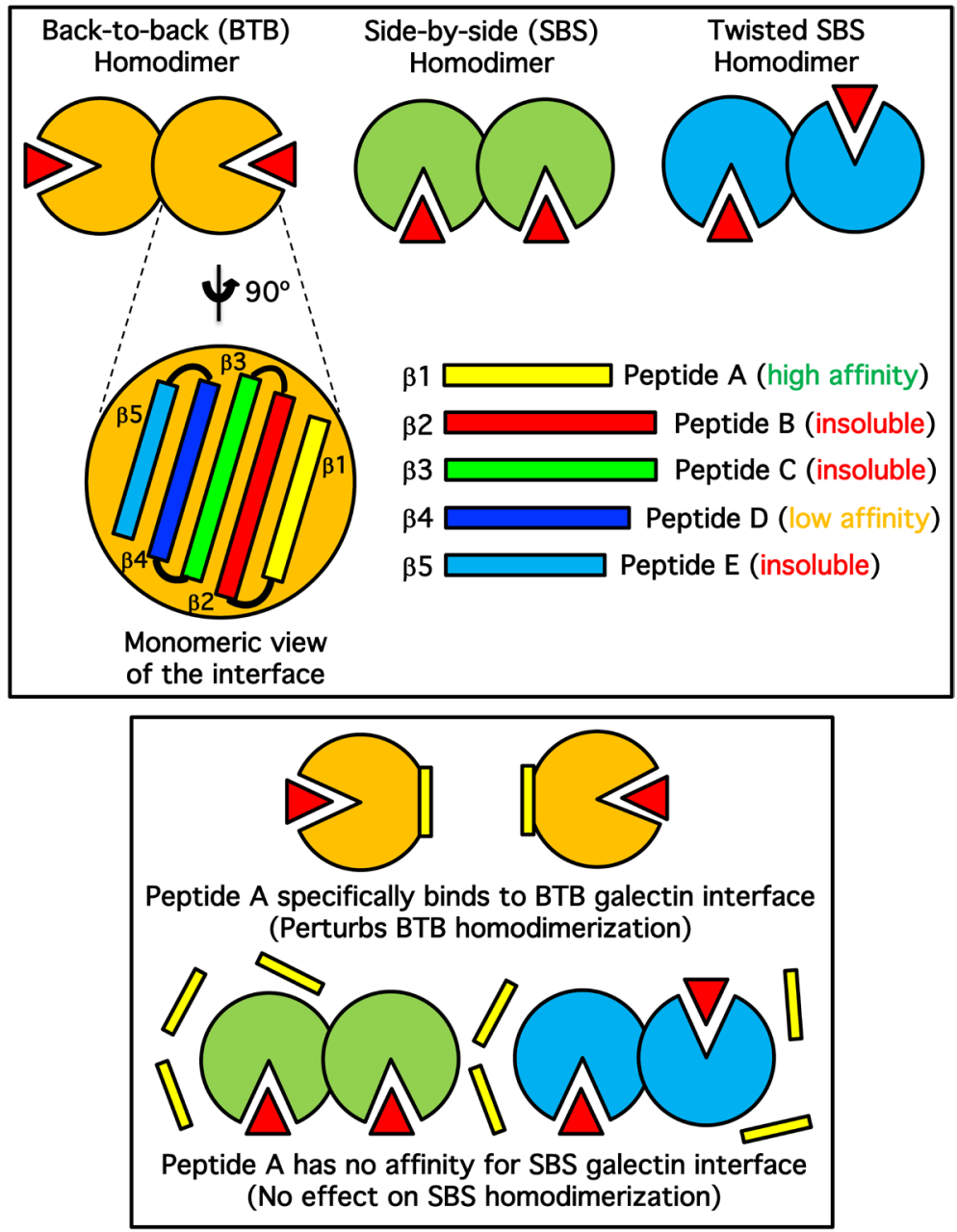

Fig. 4. Schematic illustration for designing dimer interfering peptides (DIPs) targeting a protein-protein galectin interface. Top panel: A1though sequence identity between galectin subtypes is relatively low, monomeric protomers display nearly identical 3D structures and similar binding affinities for glycosidic ligands (see Fig. 1C). This is represented by yellow, green and blue schematic protein structures that bind an identical glycoside (red triangles). In contrast, quaternary structure is specific to each galectin subtype, exhibiting various back-to-back (BTB) or side-by-side (SBS) homodimeric protein-protein architectures. Synthesis of a glycosidic inhibitor has low chance of success due to non-specific binding to the highly homologous glycan binding sites (GBS) found on all galectin subtypes (red triangles). However, the unique protein-protein interface of each galectin subtype can be rationally targeted by specific designer peptides competing with homodimeric galectin interface formation. In this case, unique molecular interactions involved in GAL-7 homodimerization were targeted by peptides corresponding to individual interface $\beta$-strands. Solubility or low affinity peptides can further be improved, namely by PEGylation, peptide mutagenesis, and/or using unnatural amino acid derivatives. Bottom panel: Synthesis of a tight DIP provides specificity against one galectin subtype, uniquely perturbing the proper homodimeric interface and only affecting the biological function of the targeted galectin. 
cancer cells (74). We and others have also reported similar findings for epithelial ovarian cancer $(75,76)$, for which there is no effective treatment. The design of a 'dimer interfering peptide' (DIP) strategy was based on the structural and rational analysis of the GAL-7 homodimeric interface, most notably targeting residues that symmetrically associate with their respective partner on the second protomer through $\mathrm{H}$-bonding, electrostatic and/or hydrophobic interactions (Fig. 3A-B). For GAL-7, DIPs were designed to rationally mimic and disrupt $\beta$-strand segments encompassing residues 13-25, 86-102, 95-108, and 129-135, since those residues are directly involved in the stabilization of the homodimeric interface (Fig. 3C). We rationalized that using these peptides to hinder the interaction with their mirror partner could effectively perturb homodimerization, further affecting the biological function of GAL-7 (Fig. 4). Our data showed that a limited number of DIPs were effective at reducing the formation of GAL-7 homodimers, but not that of GAL-1 or GAL-2, consistent with the specific signatures encoded at the dimer interface of prototypic galectins (77). Most importantly, DIPs were also effective at inhibiting GAL-7-induced apoptosis of human $\mathrm{T}$ cells. Taken together, these results suggest that the structural perturbation of GAL-7 architecture by DIPs is a promising inhibitory avenue as a new treatment for aggressive forms of cancer. This strategy also represents an ideal model for testing the specificity and potential potency of DIPs against prototypic subclasses of galectins.

\section{F. Peptides as Inhibitors of Galectin Homodimers}

While the development of DIPs to inhibit galectin homodimers is in its infancy, it can certainly take advantage of the rapidly evolving field of drugs that modulate oligomerization of protein complexes (78). The potential of targeting the homodimer/heterodimer interface as an effective means to suppress cancer is probably best illustrated by the MDM2/MDMX-p53 case (79). MDM2 is an E3 ligase that forms homodimers that bind p53, a well-known tumor-suppressor protein that functions as a transcriptional activator of many pro-apoptotic genes. Binding of MDM2 to p53 enables its E3 ligase activity to transfer ubiquitin to multiple lysine residues of $\mathrm{p} 53$, thereby negatively regulating its transcriptional activity and its tumor suppressive function. This ubiquitination is even more effective when MDM2 forms a heterodimer with MDMX, even though this protein does not harbor E3 ligase activity per se. Many investigators have thus used small molecules to disrupt MDM2/MDMX and MDM2/p53 complexes (80). Although the best-known inhibitors are probably Nutlins, a group of small cis-imidazoline analogs identified by a chemical screen (81), 12mer MDM2-inhibitory peptides (MIP) that fit in the hydrophobic pocket of MDM2 have proven to be very effective at disrupting
MDM2-p53 dimers due to their large interacting surfaces (82). The effectiveness of these 'dimer-interfering compounds' is currently being tested by several companies in phase I clinical trials, often in the context of a combined therapy with cytotoxic drugs or other drugs that target specific molecular pathways (e.g. CDK4/6, BRAF, or MEK1/2 inhibitors) (83). Other compounds are being tested alone. This is the case for DS-3022b, a drug consisting of an imidazole ring fused to a thiazole (imidazothiazoles), which is currently being tested for patients with solid tumors or lymphoma. The effectiveness of peptides to target MDM2-p53 interaction is also currently being tested in clinical trials. This is the case for ALRN6924, a stapled peptide developed by Aileron Therapeutics. Like many small drug compounds, ALRN-6924 is given orally and is currently being tested for its safety profile and its antitumor effects in patients with solid tumors or lymphoma. The use of peptides as therapeutics, most notably as anti-cancer drugs, is a rapidly growing area, most notably following the development of new technologies that render peptides suitable for oral administration and that improve their physicochemical properties. Their high sensitivity to proteolytic cleavage and their short circulating plasma half-life, two problems that have often been previously cited as important obstacles for their therapeutic use, are now minimized using various strategies (84). However, to efficiently interact with intracellular GAL-7, DIPs should retain high binding affinity, specificity, as well as high plasmatic stability, but most importantly they must be able to cross the plasma membrane. As recently demonstrated, the incorporation of an all-hydrocarbon "staple" into peptides can greatly improve pharmaceutical properties such as proteolytic stability, receptor affinity, and cell permeability (85). As such, these new derivatives, called stapled peptide, are endowed with unique and beneficial drug-like properties that solve critical problems previously plaguing the peptide class of drugs (86). The possibility of developing multifunctional peptides with dual or even triple action is another factor that favors the emergence of peptides as therapeutic agents. A case in point is the group of glucagon-like peptide-1 agonists for the treatment of type 2 diabetes mellitus [Fosgerau and Hoffmann, 2014]. These new developments certainly open up new opportunities for the use of potent and clinically relevant DIPs that will perturb the formation of galectin homodimers.

\section{G. Conclusions}

Dimer interfering peptides (DIPs) represent a new approach for the development of galectin inhibitors. Using a combination of biophysical characterization of the homodimer interface with predictive semi-rational bioinformatics approaches, we can finally envision a new generation of highly specific inhibitors capable of altering both GBS-dependent and -independent functions of pro- 
totypic galectins. The idea that tandem-repeat galectins can also form higher order aggregates that increase their avidity opens up the possibility of using DIPs for other subtypes of galectins as well [Vasta, 2012]. New DIPs can be designed on the basis of the experimental and in silico analyses of their interface and by progressive sequence optimization to improve binding affinity and specificity against their target protein. The success of this approach will certainly depend on continuous experimental and modeling feedback between scientists with distinct and complementary expertise. One can easily imagine that DIPs could also be used in the context of combinatorial therapeutic approaches using other galectin inhibitors with distinct mechanisms of action. In parallel, gaining a better knowledge of the repertoire of galectins expressed in a particular type of cancer remains critical, in addition to better defining their mode of action at different stages of tumor progression.

\section{Acknowledgments}

This work was supported in part by the Natural Sciences and Engineering Research Council of Canada (NSERC) (Discovery Grants RGPIN-2016-05557 to N.D., and RGPIN-2015-04848 to D.C.), the Fonds de la Recherche du Québec-Nature et Technologie (FRQNT) (to Y.S.P., N.D., and D.C), and the Canadian Glycomics Network (GlycoNet Translational Grant, to Y.S.P., N.D., and D.C.). N.D. holds a Fonds de Recherche Québec-Santé (FRQS) Research Scholar Junior 2 Career Award.

\section{References}

1. Vladoiu, M. C., Labrie, M., and St-Pierre, Y. (2014) Int. J. Oncol. 44, 1001-1014.

2. Rabinovich, G. A., and Conejo-García, J. R. (2016) J. Mol. Biol. 428, 3266-3281.

3. Daley, D., Mani, V. R., Mohan, N., Akkad, N., Ochi, A., Heindel, D. W., Lee, K. B., Zambirinis, C. P., Pandian, G. S., Savadkar, S., Torres-Hernandez, A., Nayak, S., Wang, D., Hundeyin, M., Diskin, B., Aykut, B., Werba, G., Barilla, R. M., Rodriguez, R., Chang, S., Gardner, L., Mahal, L. K., Ueberheide, B., and Miller, G. (2017) Nat. Med. 23, 556-567.

4. Blanchard, H., Yu, X., Collins, P. M., and Bum-Erdene, K. (2014) Expert Opin. Ther. Pat. 24, $1053-1065$.

5. Blanchard, H., Bum-Erdene, K., Bohari, M. H., and Yu, X. (2016) Expert Opin. Ther. Pat. 26, $537-554$.

6. Stannard, K. A., Collins, P. M., Ito, K., Sullivan, E. M., Scott, S. A., Gabutero, E., Darren Grice, I., Low, P., Nilsson, U. J., Leffler, H., Blanchard, H., and Ralph, S. J. (2010) Cancer Lett. 299, 95-110.

7. Ito, K., and Ralph, S. J. (2012) Clin. Exp. Metastasis 29, 561-572.

8. Glinsky, V. V., Glinsky, G. V., Rittenhouse-Olsen, K., Huflejt, M. E., Glinskii, O. V., Deutscher, S. L., and Quinn, T. P. (2001) Cancer Res. 61, 4851-4857.

9. Glinsky, G. V., Price, J. E., Glinsky, V. V., Mossine, V. V., Kiriakova, G., and Metcalf, J. B. (1996) Cancer Res. 56, 5319-5324.

10. Chauhan, D., Li, G., Podar, K., Hideshima, T., Neri, P., He, D., Mitsiades, N., Richardson, P., Chang, Y., Schindler, J., Carver, B., and Anderson, K. C. (2005) Cancer Res. 65, 8350-8358.

11. Stegmayr, J., Lepur, A., Kahl-Knutson, B., Aguilar-Moncayo, M., Klyosov, A. A., Field, R. A., Oredsson, S., Nilsson, U. J., and Leffler, H. (2016) J. Biol. Chem. 291, 13318-13334.

12. André, S., Pieters, R. J., Vrasidas, I., Kaltner, H., Kuwabara, I., Liu, F. T., Liskamp, R. M., and Gabius, H. J. (2001) ChemBioChem 2, 822-830.

13. Michel, A. K., Nangia-Makker, P., Raz, A., and Cloninger, M. J. (2014) ChemBioChem 15, $2106-2112$.

14. Cousin, J. M., and Cloninger, M. J. (2015) Beilstein J. Org. Chem. 11, 739-747.

15. Roy, R., Murphy, P. V., and Gabius, H. J. (2016) Molecules 21, E629.

16. Maljaars, C. E., André, S., Halkes, K. M., Gabius, H. J., and Kamerling, J. P. (2008) Anal. Biochem. 378, $190-196$.

17. Laderach, D. J., Gentilini, L. D., Giribaldi, L., Delgado, V. C., Nugnes, L., Croci, D. O., Al Nakouzi, N., Sacca, P., Casas, G., Mazza, O., Shipp, M. A., Vazquez, E., Chauchereau, A., Kutok, J. L., Rodig, S. J., Elola, M. T., Compagno, D., and Rabinovich, G. A. (2013) Cancer Res. 73, 86-96.

18. Grosset, A. A., Labrie, M., Vladoiu, M. C., Yousef, E. M., Gaboury, L., and St-Pierre, Y. (2016) Oncotarget 7, $18183-18203$.

19. Satelli, A., Rao, P. S., Thirumala, S., and Rao, U. S. (2011) Int. J. Cancer 129, 799-809.

20. Satelli, A., and Rao, U. S. (2011) Cancer Lett. 301, 38-46.

21. Ueda, S., Kuwabara, I., and Liu, F. T. (2004) Cancer Res. 64, 5672-5676.

22. Califice, S., Castronovo, V., Bracke, M., and van den Brûle, F. (2004) Oncogene 23, 7527-7536.

23. Saussez, S., and Kiss, R. (2006) Cell. Mol. Life Sci. 63, 686-697.

24. St-Pierre, Y., Campion, C. G., and Grosset, A. A. (2012) Front. Biosci. (Landmark Ed.) 17, 438-450.

25. Komath, S. S., Kavitha, M., and Swamy, M. J. (2006) Org. Biomol. Chem. 4, 973-988.

26. Villeneuve, C., Baricault, L., Canelle, L., Barboule, N., Racca, C., Monsarrat, B., Magnaldo, T., and Larminat, F. (2011) Mol. Biol. Cell 22, 999-1013.

27. Labrie, M., Vladoiu, M., Leclerc, B. G., Grosset, A. A., Gaboury, L., Stagg, J., and St-Pierre, Y. (2015) PLoS One 10, e0131307.

28. Shimura, T., Takenaka, Y., Tsutsumi, S., Hogan, V., Kikuchi, A., and Raz, A. (2004) Cancer Res. 64, $6363-6367$.

29. Satelli, A., Rao, P. S., Thirumala, S., and Rao, U. S. (2011) Int. J. Cancer 129, 799-809.

30. Gauthier, L., Rossi, B., Roux, F., Termine, E., and Schiff, C. (2002) Proc. Natl. Acad. Sci. U.S.A. 99, $13014-13019$.

31. Jeon, S. B., Yoon, H. J., Chang, C. Y., Koh, H. S., Jeon, S. H., and Park, E. J. (2010) J. Immunol. $185,7037-7046$.

32. Yıldırım, C., Vogel, D. Y., Hollander, M. R., Baggen, J. M., Fontijn, R. D., Nieuwenhuis, S., Haverkamp, A., de Vries, M. R., Quax, P. H., Garcia-Vallejo, J. J., van der Laan, A. M., Dijkstra, C. D., van der Pouw Kraan, T. C., van Royen, N., and Horrevoets, A. J. (2015) PLoS ONE 10, e0124347.

33. Lepur, A., Carlsson, M. C., Novak, R., Dumić, J., Nilsson, U. J., and Leffler, H. (2012) Biochim. Biophys. Acta 1820, $804-818$. 
34. Mey, A., Leffler, H., Hmama, Z., Normier, G., and Revillard, J. P. (1996) J. Immunol. 156, 1572-1577.

35. Ogden, A. T., Nunes, I., Ko, K., Wu, S., Hines, C. S., Wang, A. F., Hegde, R. S., and Lang, R. A. (1998) J. Biol. Chem. $273,28889-28896$.

36. Zhou, D., Sun, J., Zhao, W., Zhang, X., Shi, Y., Teng, M., Niu, L., Dong, Y., and Liu, P. (2006) Acta Crystallogr. Sect. F Struct. Biol. Cryst. Commun. 62, 474-476.

37. Manning, J. C., Caballero, G. G., Ruiz, F. M., Romero, A., Kaltner, H., and Gabius, H. J. (2018) Trends Glycosci. Glycotechnol. 30, SE11-SE20.

38. Ahmed, H., and Vasta, G. R. (2008) Biochem. Biophys. Res. Commun. 371, 350-355.

39. Caballero, G. G., Kaltner, H., Michalak, M., Shilova, N., Yegres, M., André, S., Ludwig, A. K., Manning, J. C., Schmidt, S., Schnölzer, M., and Bovin, N. V. (2016) Biochimie. 128, 34-47.

40. Labrie, M., Vladoiu, M., Leclerc, B. G., Grosset, A. A., Gaboury, L., Stagg, J., and St-Pierre, Y. (2015) PLoS One 10, e0131307.

41. Ermakova, E., Miller, M. C., Nesmelova, I. V., López-Merino, L., Berbís, M. A., Nesmelov, Y., Tkachev, Y. V., Lagartera, L., Daragan, V. A., André, S., Cañada, F. J., Jiménez-Barbero, J., Solís, D., Gabius, H. J., and Mayo, K. H. (2013) Glycobiology 23, 508-523.

42. Miyanishi, N., Nishi, N., Abe, H., Kashio, Y., Shinonaga, R., Nakakita, S. I., Sumiyoshi, W., Yamauchi, A., Nakamura, T., Hirashima, M., and Hirabayashi, J. (2007) Glycobiology 17, 423-432.

43. Gomes Filho, S. M., Cardoso, J. D., Anaya, K., Silva do Nascimento, E., de Lacerda, J. T., Mioso, R., Santi Gadelha, T., and de Almeida Gadelha, C. A. (2014) Molecules 20, 348-357.

44. Kamitori, S. (2018) Trends Glycosci. Glycotechnol. 30, SE41-SE50.

45. Tasumi, S., and Vasta, G. R. (2007) J. Immunol. 179, 3086-3098.

46. Feng, C., Ghosh, A., Amin, M. N., Bachvaroff, T. R., Tasumi, S., Pasek, M., Banerjee, A., Shridhar, S., Wang, L. X., Bianchet, M. A., and Vasta, G. R. (2015) Biochemistry 54, 4711-4730.

47. Gerdol, M., and Venier, P. (2015) Fish Shellfish Immunol. 46, 17-38.

48. Vasta GR, Feng C, Bianchet MA, Bachvaroff TR, Tasumi S. (2015) Fish Shellfish Immunol. 46, 94-106.

49. Luo, Y. J., Takeuchi, T., Koyanagi, R., Yamada, L., Kanda, M., Khalturina, M., Fujie, M., Yamasaki, S., Endo, K., and Satoh, N. (2015) Nat. Commun. 6, 8301.

50. Freymann, D. M., Nakamura, Y., Focia, P. J., Sakai, R., and Swanson, G. T. (2012) Acta Crystallogr. D Biol. Crystallogr. 68, $1163-1174$.

51. Ueda, T., Nakamura, Y., Smith, C. M., Copits, B. A., Inoue, A., Ojima, T., Matsunaga, S., Swanson, G. T., and Sakai, R. (2013) Glycobiology 23, $412-425$.

52. Jakób, M., Lubkowski, J., O’Keefe, B. R., and Wlodawer, A. (2015) Acta Crystallogr. F Struct. Biol. Commun. 71, 1429-1436.

53. Terada, D., Kawai, F., Noguchi, H., Unzai, S., Hasan, I., Fujii, Y., Park, S. Y., Ozeki, Y., and Tame, J. R. (2016) Sci. Rep. 6, 28344.

54. Lepur, A., Salomonsson, E., Nilsson, U. J., and Leffler, H. (2012) J. Biol. Chem. 287, 21751-21756.

55. Giudicelli, V., Lutomski, D., Lévi-Strauss, M., Bladier, D., Joubert-Caron, R., and Caron, M. (1997) Glycobiology 7, viii-X.

56. Nonaka, Y., Nakamura, T., Yoshida, H., and Kamitori, S. (2015) Photon Factory Activity Reports 2014 \#32.

57. Nonaka, Y., Ogawa, T., Yoshida, H., Shoji, H., Nishi, N., Kamitori, S., and Nakamura, T. (2015) Glycobiology 25, $792-803$.

58. Ploegh, H., and Benaroch, P. (1993) Nature 364, 16-17.

59. Schafer, P. H., Pierce, S. K., and Jardetzky, T. S. (1995) Semin. Immunol. 7, 389-398.

60. Elola, M. T., Blidner, A. G., Ferragut, F., Bracalente, C., and Rabinovich, G. A. (2015) Biochem. J. 469, 1-16.

61. Nabi, I. R., Shankar, J., and Dennis, J. W. (2015) J. Cell Sci. 128, 2213-2219.

62. Ahmad, N., Gabius, H. J., André, S., Kaltner, H., Sabesan, S., Roy, R., Liu, B., Macaluso, F., and Brewer, C. F. (2004) J. Biol. Chem. 279, 1084110847.

63. Nieminen, J., Kuno, A., Hirabayashi, J., and Sato, S. (2007) J. Biol. Chem. 282, 1374-1383.

64. Fermino, M. L., Polli, C. D., Toledo, K. A., Liu, F. T., Hsu, D. K., Roque-Barreira, M. C., Pereira-da-Silva, G., Bernardes, E. S., and HalbwachsMecarelli, L. (2011) PLoS ONE 6, e26004.

65. von Mach, T., Carlsson, M. C., Straube, T., Nilsson, U., Leffler, H., and Jacob, R. (2014) Biochem. J. 457, 107-115.

66. Roy, R., Murphy, P. V., and Gabius, H. J. (2016) Molecules 21.

67. Gitt, M. A., Wiser, M. F., Leffler, H., Herrmann, J., Xia, Y. R., Massa, S. M., Cooper, D. N., Lusis, A. J., and Barondes, S. H. (1995) J. Biol. Chem. 270, 5032-5038.

68. Barrès, C., Blanc, L., Bette-Bobillo, P., André, S., Mamoun, R., Gabius, H. J., and Vidal, M. (2010) Blood 115, 696-705.

69. Brinda, K. V., Surolia, A., and Vishveshwara, S. (2005) Biochem. J. 391, 1-15.

70. Leonidas, D. D., Vatzaki, E. H., Vorum, H., Celis, J. E., Madsen, P., and Acharya, K. R. (1998) Biochemistry 37, 13930-13940.

71. Cho, M., and Cummings, R. D. (1996) Biochemistry 35, 13081-13088.

72. Morris, S., Ahmad, N., André, S., Kaltner, H., Gabius, H. J., Brenowitz, M., and Brewer, F. (2004) Glycobiology 14, $293-300$.

73. Nesmelova, I. V., Berbís, M. Á., Miller, M. C., Cañada, F. J., André, S., Jiménez-Barbero, J., Gabius, H. J., and Mayo, K. H. (2012) Biomol. NMR Assign. 6, 127-129.

74. Demers, M., Rose, A. A., Grosset, A. A., Biron-Pain, K., Gaboury, L., Siegel, P. M., and St-Pierre, Y. (2010) Am. J. Pathol. 176, $3023-3031$.

75. Kim, H. J., Jeon, H. K., Lee, J. K., Sung, C. O., Do, I. G., Choi, C. H., Kim, T. J., Kim, B. G., Bae, D. S., and Lee, J. W. (2013) Anticancer Res. 33, $1555-1561$.

76. Labrie, M., Vladoiu, M. C., Grosset, A. A., Gaboury, L., and St-Pierre, Y. (2014) Oncotarget 5, 7705-7721.

77. Vladoiu, M. C., Labrie, M., Létourneau, M., Egesborg, P., Gagné, D., Billard, É., Grosset, A. A., Doucet, N., Chatenet, D., and St-Pierre, Y. (2015) Oncotarget 6, 40970-40980.

78. Gabizon, R., and Friedler, A. (2014) Front Chem. 2, 9.

79. Wade, M., Wang, Y. V., and Wahl, G. M. (2010) Trends Cell Biol. 20, 299-309.

80. Wade, M., and Wahl, G. M. (2009) Mol. Cancer Res. 7, 1-1.

81. Klein, C., and Vassilev, L. T. (2004) Br. J. Cancer 91, 1415-1419. 
82. Nagata, T., Shirakawa, K., Kobayashi, N., Shiheido, H., Tabata, N., Sakuma-Yonemura, Y., Horisawa, K., Katahira, M., Doi, N., and Yanagawa, H. (2014) PLoS ONE 9, e109163.

83. Burgess, A., Chia, K. M., Haupt, S., Thomas, D., Haupt, Y., and Lim, E. (2016) Front. Oncol. 6, 7.

84. Fosgerau, K., and Hoffmann, T. (2015) Drug Discov. Today 20, 122-128.

85. Verdine, G. L., and Hilinski, G. J. (2012) Methods Enzymol. 503, 3-33.

86. Jubb, H., Higueruelo, A. P., Winter, A., and Blundell, T. L. (2012) Trends Pharmacol. Sci. 33, 241-248.

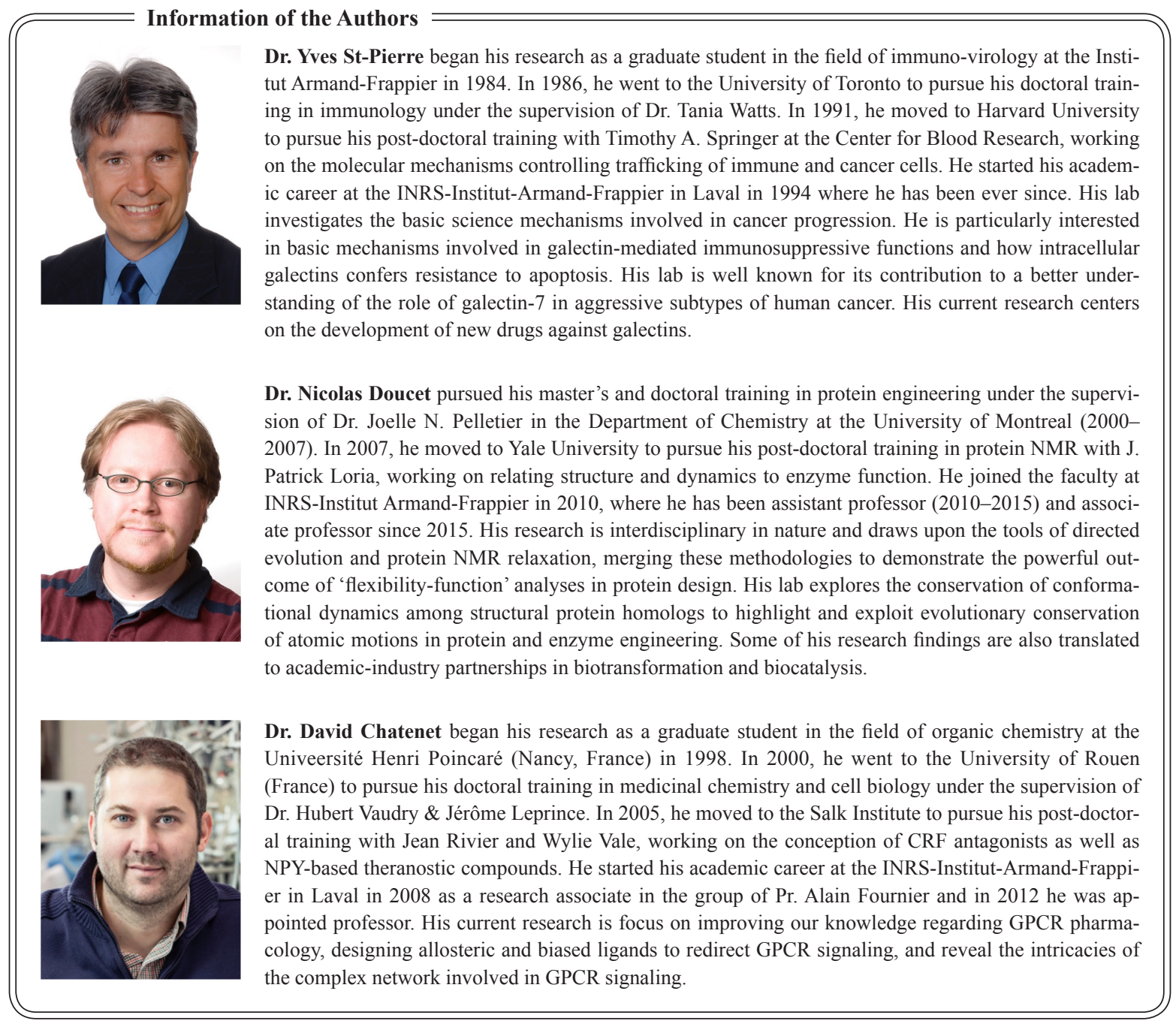

